\begin{tabular}{|l|l|l|}
\hline R. & Ralf & Babinsky $^{\mathrm{a}, \mathrm{b}}$ \\
\hline B. & Barbara & Maier $^{\mathrm{b}, \mathrm{c}}$ \\
\hline P. & Pasquale & Calabrese $^{\mathrm{a}, \mathrm{b}}$ \\
\hline H.J. & Hans J. & Markowitsch $^{\text {a }}$ \\
\hline W. & Walter & Gehlen $^{\mathrm{b}}$ \\
\hline
\end{tabular}

${ }^{\mathrm{a}}$ Physiological Psychology, University of Bielefeld, University Clinics of ${ }^{\mathrm{b}}$ Neurology and ${ }^{\mathrm{C}}$ Radiology, Knappschaftskrankenhaus Bochum-Langendreer, ${ }^{d}$ Department of Neurology, Kamillus Clinic, Asbach, Germany

\title{
Atraumatic Temporal Lobe Pathology and Autobiographical Memory
}

Ralf Babinsky, Department of Neurology, Kamillus Clinic, D-53563 Asbach (Germany)

Currently, there is an increase in reports on retrograde amnesia following lesions of more or less circumscribed brain regions. Recently, Yoneda et al. [1] reported the case of a patien suffering from encephalitis. The authors described an isolated retrograde amnesia without anterograde amnesia. The memory was impaired for the 12 months before disease onset. A SPECT study revealed left temporal lobe abnormalities, whereas CT and MRI scans were normal. Other groups found selective retrograde memory impairments in patients with traumatic head injuries [2, 3]. The lesions in these patients were centred towards anterior parts of the temporal lobes but included some additional structures. Further studies, including those on patients with a non-traumatic aetiology of brain damage, are necessary to reveal those structural correlates essential for remote memory processing.

We report anterograde and retrograde memory performance of a 64-year-old, right-handed, male patient with a non-traumatic lesion of the anterior pole of the left temporal lobe. He was admitted to hospital for developing double images and headache. Diabetes mellitus was diagnosed with an incomplete paresis of the right oculomotor nerve and a minor paresis of the left trochlear nerve due to diabetic neuropathy. Under a diet (180 g carbohydrates/day) and gli-benclamide ( $3.5 \mathrm{mg} /$ day) the symptoms disappeared, and blood sugar reached normal values within 4 days. Electro-encephalogra-phy, visual evoked and auditory evoked potentials and blink reflex were normal. CT examination revealed an arachnoid cyst at

the anterior part of the left temporal lobe (fig. 1). Further investigations showed that this malformation was liquor-filled and did not affect surrounding bones or tissue. There were no signs of space-occupying processes, and therefore the cyst was not drained or removed.

The patient was tested neuropsychologi-cally 15 days after arriving at the hospital. At this time, the neuropathies had faded away, and the patient's behaviour during the examination was unremarkable and appropriate. Several tests to examine attention, intelligence, affective state, concept comprehension and anterograde as well as retrograde memory functions were carried out.

A German Personality Inventory gave no remarkable results, in particular no signs of depression or some other psychological/psychiatric abnormalities. Intellectual abilities were above average (IQ 121, reduced Wechsler Adult Intelligence Scale [4]) and a Concept Comprehension Task [5] revealed no deficits (concrete concepts 8/8; abstract concepts 7/8). Performance in attention tests, measuring concentration (Concentration Endurance Test d2 [6]) and information-processing speed (a German version of the Trail-Making Test A [7]), was within normal limits (around the 50th percentile). His immediate memory span was normal (digit span: 6; Corsi block span: 5), and the Wechsler Memory Scale (WMS-R [8]) showed that anterograde and retrograde (up to $30 \mathrm{~min}$ ) memory performance was at or above average (general memory: 126; delayed recall: 113). In a subtest of a German aphasia test he also showed no naming defi-

no naming defifrom 1938 to 19 90) and personal semantic memory [9] (facts remembered: childhood 74\%, early adult life $100 \%$, recent life $91 \%$ ) on the one hand and definitely subnormal autobiographical episodic memory [9] for the last 10 years on the other hand (remembered episodes: childhood 100\%, early adult life $100 \%$, recent life $56 \%$ ).

To our knowledge, the present case is the first with neuroradiologically described nontraumatic pathology ofthe left temporal pole and a test-verified autobiographical memory impairment. The study of Yoneda et al. [1] underscores the significance of left temporal lobe structures for retrograde memory processing. Other cases showed severer pathologies which extended beyond both temporal lobes [2, 3] but were centred to anterior parts of the temporal lobes, too. One study by De Renzi et al. [10] showed some different results. A patient with lesions in several areas of the inferior and anterior part of the left temporal lobe (involving the amygdala, hippocampus and surrounding structures) showed large deficits in non-personal episodic memory but had preserved autobiographical memory. Regarding the extensive lesions in the latter case, we conclude from our case with a circumscribed lesion and a circumscribed memory deficit that anterior parts of the left temporal lobe may be involved in the effortful processing of episodic autobiographical memories and therefore of uniquely stored personal incidents. 


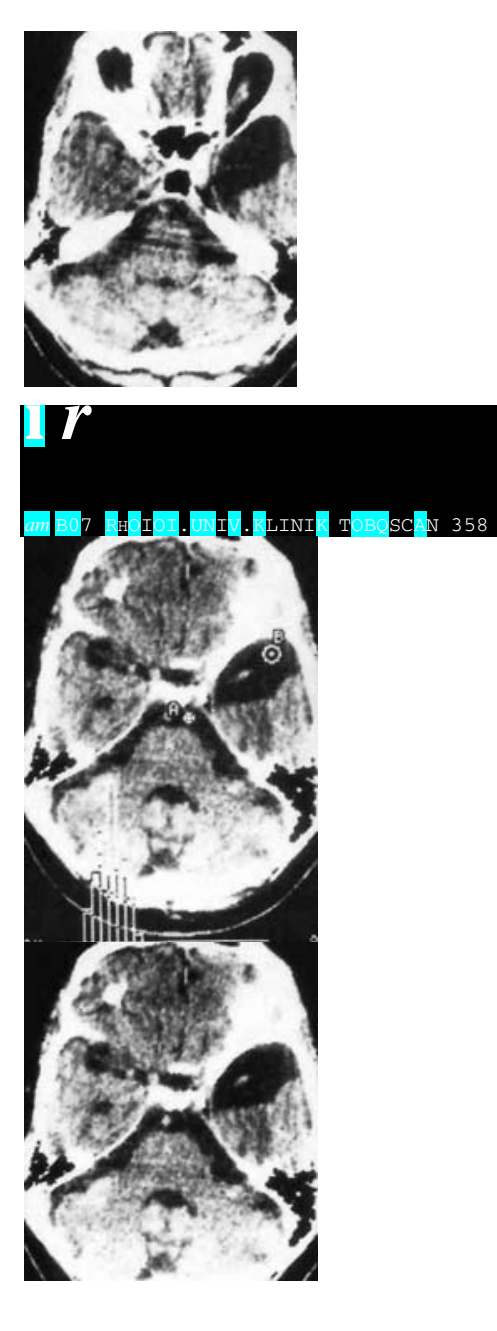

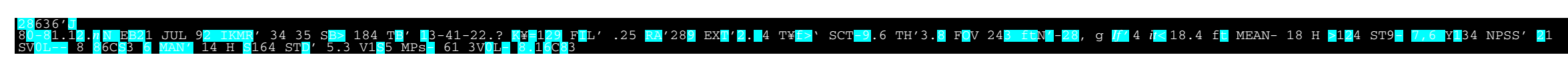

Fig. 1. CT scan showing the arachnoid cyst of the patient. Upper left: this special scan revealed no 'watch-glass phenomenon' and therefore proved the absence of pressure effects. Upper right: horizontal CT scan at the same level (internal auditory canal) as the upper left scan, showing the arachnoid cyst at the left temporal pole. Bottom left and right: CT scans (at the level of the pons) and data from measuring the optic density of two areas - the arachnoid cyst and a ventricle - showing comparable results (approx. 10-14 Hounsfield units, with overlapping SD), revealing that both parts were filled by the same fluid, probably cerebrospinal fluid.

References 1 Yoneda Y, Yamadori A, Mori E, Yamashita H:

2 Kapur N, Ellison D, Smith MP, McLellan DL, Burrows EH: Focal retrograde amnesia following bilateral temporal lobe pathology. Brain 1992;115:73-85.

3 Markowitsch HJ, Calabrese P, Haupts M, Durwen HF, Liess J, Gehlen W: Searching for the anatomical basis of retrograde amnesia. J Clin Exp Neuropsychol 1993;15:947-967.

4 Dahl G: WIIP - Reduzierter Wechsler-Intelli-genztest. Meisenheim, Hain, 1986. 
Brickenkamp R: Test d2, Aufmerksamkeits-Bellastungs-Test. Gottingen, Hogrefe, 1981. Oswald WD, Roth E: Zahlen-Verbịndungs-Test ZVT. Gottingen, Hogrefe, 1987. Wechsler D: Wechsler Memory Scale Revised. New York, Psychological Corporation, 1987.

Kopelman MD, Wilson BA, Baddeley AD: The Autobiographical Memory Interview: A new assessment of autobiographical and personal semantic memory in amnesic patients. J Clin Exp Neuropsychol 1989; 11:724-744. De Renzi E, Liotti M, Nichelli P: Semantic amnesia with preservation of autobiographical memory - A case report. Cortex 1987;23:575-597. 